Journal of ISMAC (2020)

Vol.02/ No. 01

Pages: 62- 72

http://irojournals.com/iroismac/

DOI: https://doi.org/10.36548/jismac.2020.1.006

\title{
A Novel Multi-Tier Architecture Based Mobile Cloud Computing For Enhanced Energy Utilization
}

\author{
Prof. Sathish, \\ Department of EEE, Eritrea \\ Institute of Technology, Eritrea. \\ Email: sathesh4you@gmail.com \\ Dr. S. Smys, \\ Professor, Department of Computer Science and Engineering, \\ RVS Technical Campus, \\ Coimbatore, India. \\ Email: smys375@gmail.com
}

\begin{abstract}
The recent advancements in the mobile devices and the growing needs of the mobile users led to the clubbing of mobile devices with the cloud computing developing a platform coined as mobile cloud computing. The main scope of conceiving this clubbed mobile and cloud paradigm is to direct the tasks that are resource and computationally intensive to the cloud for its execution. While the execution takes place in the cloud, the resources of the mobile users that are clubbed with the cloud remains wasted until the responses are received from the cloud. These results in the excess battery drain causing frequent recharges. To put an end to this, the paper puts forward an the multi-tier architectures in-built with the various level of cluster process for processing to properly handle the mobile device participating in the cloud, minimizing the number of idle mobile users and enhances the energy efficiency. The proposed method is validated using the network simulator-II to evince the energy balancing achieved through the proposed multi-tier architecture based MCC-(mobile cloud computing).
\end{abstract}

Keywords: Cloud Computing, Mobile devices, Mobile Cloud Computing, Multi -tier architecture and Energy Efficiency

\section{Introduction}

Advancements in the mobile devices have made them the inevitable component in the day to day activities of the humans. The advancements pave way for the growing demands from the humans leading to the accessing of more and more application that are resource and computationally intensive. This was the reason to develop a paradigm that clubbed both the mobile devices and the cloud computing. The structure

ISSN: 2582-1369 (online) 
Journal of ISMAC (2020)

Vol.02/ No. 01

Pages: 62- 72

http://irojournals.com/iroismac/

DOI: https://doi.org/10.36548/jismac.2020.1.006

of the MCC is architecture with the, mobile devices, the system to enable the vice versa communication and the cloud frame work. The figure. 1 is the frame of MCC that combines both the cloud and the mobile devices to enhance the performance of the mobile applications.

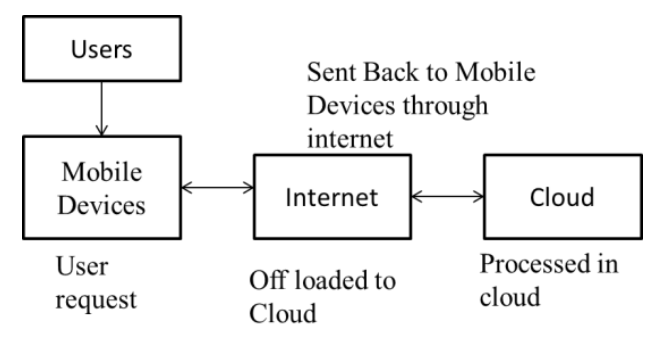

Figure.1 Mobile Cloud Computing Paradigm

The Mobile service integrated with the cloud is almost applicable in many applications used in rural as well as urban areas. It accomplishes a complicated connection between the providers of the infrastructure, service and applications and also the developers and the users at the end who are none other than the mobile users at the end. The infrastructure, the service and the application providers are the stake holders in the cloud providing the required hardware and the software requirements as per the request, the execution of the services respectively whereas the developers the consumers of the cloud who develop the required applications that are hosted on the datacenters of the cloud.

This mobile paradigm including the cloud services and the complicated connections between the providers of the services as stated above totally relies proper clubbing of the infrastructures that is both hardware and software. But still there are certain issues that cause practical challenges in managing the energy utilization of the mobile integrated cloud paradigm.

The main scope of the integrating the mobile with the cloud is to elude the performance deterioration of the mobile devices due to the low storage and processing and the transmission limitations, to overcome this the mobile device offloaded their computationally intensive task with dynamic characteristics to the cloud paradigm that is rich in resources, storage, computational capabilities and available at the limited cost with the pay as you go terms. In most cases the offloading is done to an external cloud where processing and storage is done outside the phone and in certain case the mobile devices with the support of cloud does the

ISSN: 2582-1369 (online) 
Journal of ISMAC (2020)

Vol.02/ No. 01

Pages: 62- 72

http://irojournals.com/iroismac/

DOI: https://doi.org/10.36548/jismac.2020.1.006

processing and as well as storage. Two ways of MCC implementation strategies that are followed till now are the static hardware infrastructure provided by the cloud and the other one is the volunteering mobile devices networked in adhoc manner to share the resources as well as the processing capacities of the mobile devices in the network. Offloading the process completely to the cloud and usage of static hardware infrastructure is necessitated only when the demands of the computation goes beyond the capabilities of the mobile devices. But a mobile integrated cloud service model at least should have a partial participation in the processing rather than totally relying on the datacenters. The infrastructure that helps in extending the communication between the cloud backend servers and the mobile devices impacts more on the complete energy utilized.

Many methodologies such as the opportunistic offloading, avalanche offloading, and the middleware based scheduling of tasks and multiple schemes to conserve the energy utilization were developed in the recent years, but were not much convincing as they emphasize only on conserving the mobile resources rather than properly utilizing it and leaned on the efficiency of the cloud eluding the solution that would enhance the energy efficiency. The mobile devices that offload completely to the cloud stay idle until the response is received so the waiting time and the delay in the processing affect the overall energy efficiency. So as to improve the energy efficiency the proposed process puts forth methods to make the mobile devices participate in the execution instead of staying idle, and minimize the number of mobile device in idle turn minimizing the overall energy utilization.

So the paper develops a processing multi-tier structure that is built using various mobile clusters with varying capacities networked in adhoc manner to perform an execution that with enhanced energy utilization. The paper holds the literature survey in its $2^{\text {nd }}$ section, the proposed work in the $3^{\text {rd }}$ section, the performance analysis in the $4^{\text {th }}$ section followed by the conclusion in the $5^{\text {th }}$ section.

\section{Literature survey}

The data computation and the storage process done in the cloud for the mobile device consumes high energy due to the delay incurred in the processing of the tasks. To ignore this problem many researches have done to devise a proper frame work that effectively manages the resources to minimize the consumed energy. The literature survey highlights few similar works that scope to reduce the energy consumption in the MCC. The author Liu, et al [1] has framed a task offloading strategy with the help of the self-organized criticality proposing an algorithm to save energy by reducing the delay in the services. Nir et al [2] devises an energy

ISSN: 2582-1369 (online) 
Journal of ISMAC (2020)

Vol.02/ No. 01

Pages: 62- 72

http://irojournals.com/iroismac/

DOI: https://doi.org/10.36548/jismac.2020.1.006

consumption model by putting into action the a "centralized broker that optimally offloads the tasks and provides significant minimization of energy" Li, et al [3] the author develops an "ENDA" architecture with three tier and provides an optimized decision for the offloading of the mobile services to cloud considering the performance of the network and the track prediction. Kumar, T. Senthil et al [4] has put forth the resource allocation scheme to enhance the quality of the service in the internet of things,

Gao, Wei. et al [5] develops an adaptive method exploiting the mobile devices to configure the computational work load allocations among the mobile devices. Taking into consideration the both the energy consumption and the time taken for executing the tasks. Opportunistic transmission is followed in the paper to minimize the energy consumption. Liu, et al [6] puts forth the concept of resource sharing mechanism in the mobile devices with the help of contacts that are opportunistic in order to minimize the latencies in the computation. Min, et al [7] has put forth a novel framework termed E2S3, to make effective sleep state selection to bring down the power consumed and extend the battery life without compromising the system performance. Bhalaji, $\mathrm{N}$ et al [8] proposes a "delay diminished task scheduling for the heterogeneous cloud services". Xia, et al [9] proposes the computational offloading method to improve the energy efficiency as well as the execution time so as to enhance the experience of the user. Bashar et al [10] puts forth the" secure cost efficient offloading technique for the mobile computing" Gao et al [11] the paper "investigates the possibility of developing energy-aware offloading strategies for workflows" Zhang, et al [12] develops an ""Energy-efficient scheduling policy for collaborative execution in mobile cloud computing." and Shu, et al [13] puts forth the "eTime: Energy-efficient transmission between cloud and mobile devices." the author Kumar, et al [14] elaborates the "Review on task scheduling in ubiquitous clouds."

Form the survey the Basic challenges in improving the energy efficiency in the Cloud based mobile architecture is presented in the table. 1 below.

\begin{tabular}{|l|l|}
\hline Challenges incurred in MCC & \multicolumn{1}{c|}{ Description } \\
\hline Offloading to Distant cloud & Causes delay in propagation and transmission \\
\hline Network Latency & $\begin{array}{l}\text { Degrades the communication and the system } \\
\text { responses }\end{array}$ \\
\hline $\begin{array}{l}\text { Distance between the terminals of the mobile } \\
\text { devices }\end{array}$ & Develops dead spots and coverage holes \\
\hline
\end{tabular}

ISSN: 2582-1369 (online) 
Journal of ISMAC (2020)

Vol.02/ No. 01

Pages: 62- 72

http://irojournals.com/iroismac/

DOI: https://doi.org/10.36548/jismac.2020.1.006

\begin{tabular}{|l|l|}
\hline Energy cost & Increases due to latencies incurred. \\
\hline Device Heterogeneity & Causes vendor lock- in \\
\hline
\end{tabular}

Table.1 Basic Challenges in MCC

\section{Novel Multi-tier Frame Work for the Cloud Integrated Mobile Device}

The multi-tier frame work that is developed to bring down the energy consumption and utilizes majority of mobile devices that stay idle during the processing is composed of both cloud paradigm and the mobile devices connected in the adhoc manner which constitutes four different groups devices networked in adhoc manner with varying capacities they are innate mobile station, the simple network framed with the volunteering mobile devices in a multi-tier adhoc manner, the cloud data centers cluster to manage the heavy tasks, and finally the combined architecture that integrates both the cloud and the mobile cluster, which also known as hybrid cluster is included to the architecture to enable a computing that is energy efficient by utilizing both the resources of the cloud and the mobile devices. The figure. 2 below provides the architecture for the proposed.

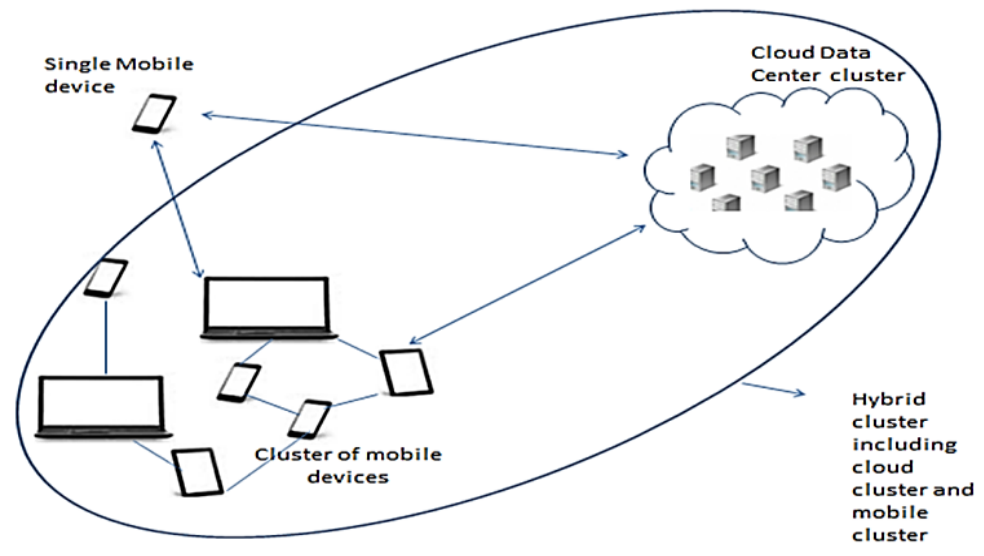

Figure.2 Proposed Architecture

ISSN: 2582-1369 (online) 
Journal of ISMAC (2020)

Vol.02/ No. 01

Pages: 62- 72

http://irojournals.com/iroismac/

DOI: https://doi.org/10.36548/jismac.2020.1.006

The proposed architecture fixes couple of devices $\left\{D_{M}, D_{S}\right\}$ to completely monitor the network activities such that no resources are left idle and all the resources engaged either takes the role of consumer or provider and not just the service-requester alone. The resources that are available in the Cloud based Mobile are considered under the $D_{S}$, whereas the $D_{M}$, is employed to manage proper resource allocation. The particulars such as the speed of processing $(P S d)$, the available memory space ( $M S a)$, the residual energy $(R E)$ of the battery for the each device, the total number of slots $(T S l)$ volunteered by the mobile devices, and the available time $\operatorname{slot}\left(\operatorname{Ts} a\right.$ ) in the mobile devices that are assigned under the $D_{s}$ are gathered and the $D_{M}$, takes care of locating the volunteering devices. The availability of the slots under the $D_{S}$ is enumerated using the equation give below.

$$
A_{\text {Dsslots }}=\sum_{x=1}^{n} s_{a}^{t_{j}} T_{\text {prob } t(j)-t(j+1)}
$$

Where $s_{a}^{t_{j}}$ is the availability of slot' a' at time $t_{j}$ and $T_{\text {prob } t(j)-t(j+1)}$ is the probability of transition from $t(j)$ tot $(j+1)$. To reduce the delay in the transmitting the tasks from one cluster to the next the tasks are clustered according to their requirements, such as number of $T S l$ required $\left(T S I_{R}\right)$, the $M S a$ required $\left(M S a_{R}\right)$, The deadline of the task $\left(T_{d l}\right)$, so the number tasks that could be computed in the totally relies on the available resources and the expectations of the user. So according to the expectation of the user and deadline assigned to the task, the resources usage could be increased or decreased. so the resources available are clustered according to the requirements put forth by the user.

The algorithm below in the figure. 3 depicts the energy efficient management of the resources according to the task requisitions.

Based on the algorithm given above the proposed architecture starts executing, the received requests. On receiving the requisition from the users the $D_{M}$, tries to identify the compatible device locally and if failed extends a search by generating a requisition among the nearby devices upgrading the communication medium in multi-tier fashion from Bluetooth to Wi-Fi and Wi-Fi to cellular network, and identifies the suitable devices, this meanwhile the $D_{S}$ checks for the capabilities of the resources and confirms the $D_{M}$, about the resource suitability to the task. The $D_{M}$, on its failure in identifying the suitable resource hand overs the requisition to the cloud data centers for processing. The proposed method removes and rejoins the devices to the cluster based on the user requirement to reduce the number of idle devices.

ISSN: 2582-1369 (online) 
Journal of ISMAC (2020)

Vol.02/ No. 01

Pages: 62- 72

http://irojournals.com/iroismac/

DOI: https://doi.org/10.36548/jismac.2020.1.006

Input: Resource Capacity PSd, RE, TSl, MSa,Tsa

Task Requirement $T S I_{R}, M S a_{R}, T_{d l}$

Output: : Proper resource allocation

i. Gather Task Requirements (Task ${ }_{\text {req }}$ ) for the requisition received

ii. For each mobile device

iii. Enumerate Resource capacity (Res cap $_{\text {) }}$ )

iv. If $\left(\right.$ Task $_{\text {req }}<$ Res $\left._{\text {cap }}\right) / /$ of the particular mobile device

v. Execute task in the mobile device itself

Else

vi. If $\left(\right.$ Task $_{\text {req }}>$ Res $\left._{\text {cap }}\right)$

vii. Triggers request to nearby mobile devices //Using the Bluetooth

viii. If identified

ix. Add devices to the cluster goes to step iii

Else

Triggers request to nearby mobile devices //using Wi-Fi

if Identified Goes to ix

Else

Triggers request to nearby mobile devices// using Cellular Network if Identified Goes to ix

Else

Transmits the task to the cloud data centers.

X. If $\left(\right.$ Res $_{\text {cap }} \gg$ Task $\left._{\text {req }}\right)$

Enumerating Res cap $_{\text {pa }}$

If $\left(\right.$ Task $_{\text {req }}=$ Res $\left._{\text {cap }}\right)$

Executes task

Else

Removes the mobile devices

Goes to step X.

Figure.3 Proposed Resource Allocation based on Task Requirement

ISSN: 2582-1369 (online) 
Journal of ISMAC (2020)

Vol.02/ No. 01

Pages: 62- 72

http://irojournals.com/iroismac/

DOI: https://doi.org/10.36548/jismac.2020.1.006

\section{Performance Evaluation}

The proposed framework is evaluated using the network simulator-2 for different number of tasks, different types of mobile devices with varying capabilities, and Communication medium. The table. 2 below lists the details of the resources that were employed in the proposed architecture.

\begin{tabular}{|l|l|}
\hline \multicolumn{1}{|c|}{ Parameters } & \multicolumn{1}{c|}{ Values } \\
\hline Number of Tasks & $50,100,150,200,250,300,350,400,450,500$ \\
\hline Types of mobile devices & 3 (mobile phone, lap top and tablet) \\
\hline Types of Communication Medium & Bluetooth, Wi-Fi, Cellular Network \\
\hline Processing power of devices & Ranging From (20 mW-360 W) \\
\hline Processing power of Access technology & Ranges From (.01- 1watts ) \\
\hline Simulation period & $2500 \mathrm{~ms}$ \\
& \\
\hline
\end{tabular}

Table.2 Parameters Involved

The results were evaluated based on the time taken for the processing and the energy consumed during the processing for different number of tasks. The figure. 4 below is the results observed on the total power consumed on executing a single task using either the mobile device itself or a local mobile device or a cloud data center, the average power consumed for an execution of the task involves the power required to transmit, transit, process, return and receive, So the power requirement increases based on the requirement of the tasks, and the capabilities of the device, if the task requirement is low and the capability of the local device is high the power consumed is low and if the task requirement is high and the capability of the local device is low then the power consumption increases, as it involves the request generation, device capability identification and communication medium up gradation etc.

ISSN: 2582-1369 (online) 
Journal of ISMAC (2020)

Vol.02/ No. 01

Pages: 62- 72

http://irojournals.com/iroismac/

DOI: https://doi.org/10.36548/jismac.2020.1.006

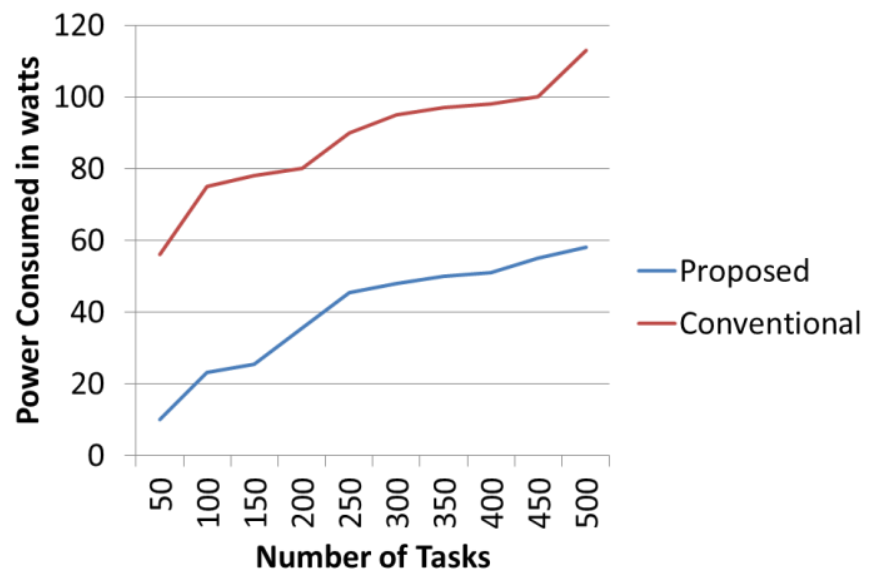

Figure.4 Power Consumed

The results observed shows that the power consumption is found be low for the local mobile device that are communicated employing the Bluetooth and goes on increasing as the communication medium also evolves, yet found to manage the resource allocation effectively with proper energy utilization compared to the conventional methods.

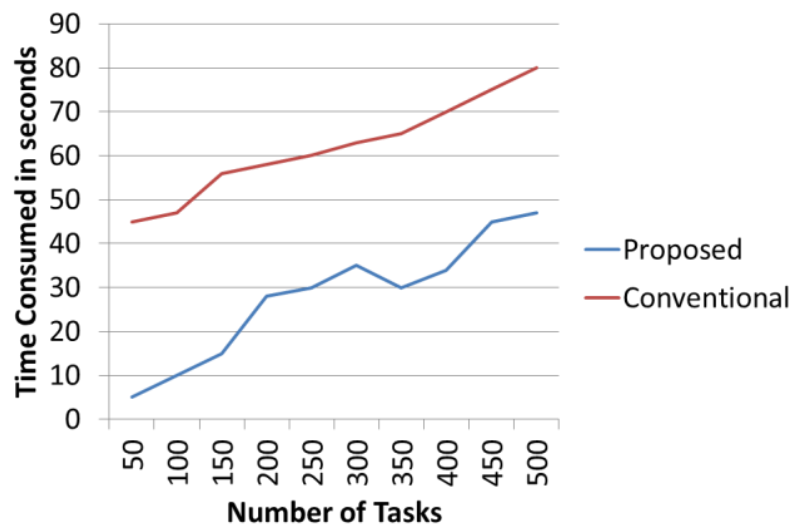

Figure.5 Total Time consumed

ISSN: 2582-1369 (online) 
Journal of ISMAC (2020)

Vol.02/ No. 01

Pages: 62- 72

http://irojournals.com/iroismac/

DOI: https://doi.org/10.36548/jismac.2020.1.006

The figure .5 is the results observed on the total time taken to transit out, transit in and process the tasks, with the different varieties of resources utilized, this totally depends on the capacities of the devices and the requirements of the tasks the results obtained for the number of tasks proved that the proposed out performs the conventional by consuming considerable time for processing and transmitting and receiving.

\section{Conclusion}

The proposed architecture aims in minimizing the idle resources while offloading the service requisitions from the mobile to the cloud and reduces the overall energy consumed. For effective management the proposed method gathers the requirements of the tasks and clusters the resources according to the task requirement by removing the excess resources and adding the resources if necessary, this helps in reducing the idle resources and more over the proper management of task execution identifying the perfect compatible nearby resources upgrading the communication medium in the multitier fashion ensures the fastest processing with the minimized execution time and power consumption. The results obtained by evaluating the proposed method proves that the proposed method of resource allocation in MCC is outperforms the conventional resources allocation procedure followed in the $\mathrm{MCC}$ in terms of computation time and power.

\section{References}

[1] Liu, Xing, Chaowei Yuan, Zhen Yang, and Zhongwei Hu. "An energy saving algorithm based on user-provided resources in mobile cloud computing." In 2013 IEEE 78th Vehicular Technology Conference (VTC Fall), pp. 1-5. IEEE, 2013.

[2] Nir, Manjinder, Ashraf Matrawy, and Marc St-Hilaire. "An energy optimizing scheduler for mobile cloud computing environments." In 2014 IEEE Conference on Computer Communications Workshops (INFOCOM WKSHPS), pp. 404-409. IEEE, 2014.

[3] Li, Jiwei, Kai Bu, Xuan Liu, and Bin Xiao. "Enda: Embracing network inconsistency for dynamic application offloading in mobile cloud computing." In Proceedings of the second ACM SIGCOMM workshop on Mobile cloud computing, pp. 39-44. 2013.

[4] Kumar, T. Senthil. "Efficient resource allocation and QOS enhancements of IoT with FOG network." J ISMAC 1 (2019): 101-110.

[5] Gao, Wei. "Opportunistic peer-to-peer mobile cloud computing at the tactical edge." In 2014 IEEE Military Communications Conference, pp. 1614-1620. IEEE, 2014. 
Journal of ISMAC (2020)

Vol.02/ No. 01

Pages: 62- 72

http://irojournals.com/iroismac/

DOI: https://doi.org/10.36548/jismac.2020.1.006

[6] Liu, Wei, Ryoichi Shinkuma, and Tatsuro Takahashi. "Opportunistic resource sharing in mobile cloud computing: The single-copy case." In The 16th Asia-Pacific Network Operations and Management Symposium, pp. 1-6. IEEE, 2014.

[7] Min, Alexander W., Ren Wang, James Tsai, Mesut A. Ergin, and Tsung-Yuan Charlie Tai. "Improving energy efficiency for mobile platforms by exploiting low-power sleep states." In Proceedings of the 9th conference on Computing Frontiers, pp. 133-142. 2012.

[8] Bhalaji, N. "Delay Diminished Efficient Task Scheduling And Allocation For Heterogeneous Cloud Environment." Journal of trends in Computer Science and Smart technology (TCSST) 1, no. 01 (2019): 51-62.

[9] Xia, Feng, Fangwei Ding, Jie Li, Xiangjie Kong, Laurence T. Yang, and Jianhua Ma. "Phone2Cloud: Exploiting computation offloading for energy saving on smartphones in mobile cloud computing." Information Systems Frontiers 16, no. 1 (2014): 95-111.

[10] Bashar, Abul. "Secure And Cost Efficient Implementation Of The Mobile Computing Using Offloading Technique." Journal of Information Technology 1, no. 01 (2019): 48-57.

[11] Gao, Bo, Ligang He, Limin Liu, Kenli Li, and Stephen A. Jarvis. "From mobiles to clouds: Developing energy-aware offloading strategies for workflows." In 2012 ACM/IEEE 13th International Conference on Grid Computing, pp. 139-146. IEEE, 2012.

[12] Zhang, Weiwen, Yonggang Wen, and Dapeng Oliver Wu. "Energy-efficient scheduling policy for collaborative execution in mobile cloud computing." In 2013 Proceedings Ieee Infocom, pp. 190-194. IEEE, 2013.

[13] Shu, Peng, Fangming Liu, Hai Jin, Min Chen, Feng Wen, Yupeng Qu, and Bo Li. "eTime: Energy-efficient transmission between cloud and mobile devices." In 2013 Proceedings IEEE INFOCOM, pp. 195-199. IEEE, 2013.

[14] Kumar, Dinesh. "Review on task scheduling in ubiquitous clouds." J. ISMAC 1, no. 01 (2019): $72-80$.

ISSN: 2582-1369 (online) 